Bull. Mater. Sci., Vol. 17, No. 6, November 1994, pp. 771-781, (O) Printed in India.

\title{
Formation and propagation of bands in jerky flow: a coupled lattice map description*
}

\author{
G ANANTHAKRISHNA \\ Materials Research Centre, Indian Institute of Science, Bangalore 560012 , India.
}

\begin{abstract}
There has been revival of interest in Jerky flow from the point of view of dynamical systems. The earliest attempt in this direction was from our group. One of the predictions of the theory is that Jerky flow could be chaotic. This has been recently verified by us. We have recently extended the earlier model to account for the spatial aspect as well. Both these models are in the form of coupled set of nonlinear differential equations and hence, they are complicated in their structure. For this reason we wish to devise a model based on the results of these two theories in the form of coupled lattice map for the description of the formation and propagation of dislocation bands. We report here one such model and its results.
\end{abstract}

Keywords. Jerky flow; Portevin-Le Chatelier effect; chaos; coupled lattice maps.

\section{Introduction}

Different kinds of instabilities manifest in plastic flow experiments depending upon the mode of the experimental set up. Jerky flow or the Portevin-Le Chatelier effect (PLC) arises in a constant strain rate experiment. Although, this has been recognized as some kind of instability for a long time, it is only in the last decade that it has been analyzed from the point of dynamical systems. It has been long known that Jerky flow arises from the dynamic interaction of dislocations and mobile point defects and is referred to as dynamic strain ageing (DSA). It is this that induces a negative strain rate sensitivity (SRS) which in turn triggers the instability. Even so, several aspects of the phenomenon were not well understood until recently. In the past few years, there has been renewed attempts (Ananthakrishna and Sahoo 1981; Ananthakrishna and Valsakumar 1982; Valsakumar and Ananthakrishna 1983; Kubin and Estrin 1985, 1990; Zbib and Aifantis 1988; Jeanclaude and Fressengeas 1993; See also the papers in Viewpoint set 1993) to understand the phenomenon from the point of view of the theory of dynamical systems (Berge et al 1984; Hao Bai-lin 1988, 1989, 1990). The essential content of most of these studies is to re-examine all aspects of PLC in the light of the intrinsic nonlinear nature of the phenomenon. This has helped to get new insights hitherto not possible. All these theories emphasize the intrinsic nonlinearity inherent in the phenomenon. Of course, the details and levels of description of the phenomenon are different. One of the aims of such theories is to relate the microscopic dislocation mechanisms to the macroscopic measurable quantities. Even in such theories which stress the dynamical basis of the PLC effect, the negative SRS is an input in one form or

*The material contained here was a part of the Plenary Lecture presented at the International Conference on Plasticity of Materials: Fundamental Aspects of Dislocation Interactions, Ascona, Switzerland, 1992. 
the other with one notable exception of a model introduced by us. This attempts to derive all results as a consequence of the nonlinear interaction between the various types of dislocations (Ananthakrishna and Sahoo 1981; Ananthakrishna and Valsakumar 1982, 1983; Valsakumar and Ananthakrishna 1983; Ananthakrishna 1992a), including the negative SRS. For this reason, this model is completely dynamic in character. The method involves setting up the time evolution equations for the dislocation densities. The negative SRS results as a consequence of Hopf bifurcation from time homogeneous steady state to the time oscillatory state. Although, this theory ignored the spatial aspects, it proved to be surprisingly successful in that it could explain several features of the Jerky flow, such as the existence of a window of strain rates, temperature and solute concentration over which the phenomenon is seen, and the emergence of the negative SRS. One of the predictions of the model is that there is a range of values of applied strain rate where the plastic flow is chaotic (Ananthakrishna and Valsakumar 1983; Ananthakrishna 1990, 1992a). Recently, this prediction has been verified by anlyzing the experimental signals from two distinct groups (Ananthakrishna 1992, 1994; Ananthakrishna 1993). It must be remembered that the experimental signals correspond to spatially extended system, and therefore, the existence of a strange attractor for the experimental signals with a fractal dimension $d_{f}$ implies that $d=2 d_{f}+1$ degrees of freedom correspond to collective degrees of freedom of the spatially extended system. This in turn means that the dynamical basis of the model viz. that there are only few dynamical degrees of freedom for the phenomenon is indeed correct.

One controversial aspect of the Jerky flow is devising an appropriate framework for including the spatial dependence (Kubin and Estrin 1985, 1990; Zbib and Aifantis 1988; Jeanclaude and Fressengeas 1993; Ananthakrishna 1993; See the papers in Viewpoint set 1993). There are several approaches which can be broadly classified as reaction-diffusion schemes. They largely depend on either long range dislocation interactions or the cross slip mechanism. In most cases, the negative SRS is an input into diffusion like equations (Zbib and Aifantis 1988; Jeanclaude and Fressengeas 1993; Kubin et al 1993). In contrast to these models, we have extended the above dynamical model by writing continuity equations which explain several features of the formation and propagation of the dislocation bands (Ananthakrishna 1993). The state of art of the subject is well summarized by the Viewpoint set of papers (Kubin et al 1993). Both these models are complicated in nature and hence not very transparent. The purpose of this paper is to present an alternate way to model the spatial aspects of the PLC effect on the basis of the predictions of both these dynamical models. One standard way to handle spatially extended dynamical system when individual elements are chaotic is to set up coupled lattice maps (Kaneko 1989). This will be done using the fact that the model predicts chaos and the one dimensional maps associated with the set of differential equations of the model are quadratic in nature (Feigenbaum 1978; Ananthakrishna and Valsakumar 1983; Ananthakrishna 1990, 1992a). We will attempt to provide arguments in support of such modelling. The plan of the paper is as follows. In $\S 2$, we will very briefly recall the results relevant for building the coupled map lattice model. The model presented in $\$ \S 3$ and 4 contains results of the model. We end the paper by some remarks on the utility of such models. 


\section{Chaotic behaviour of the dynamical model for the Jerky now}

In the following we will very briefly recall some relevant results of the model and make use of the results in setting up a coupled lattice map model. The dynamical model consists of three types of dislocations, viz. the mobile dislocations, the immobile dislocations, another type which may be regarded as dislocations with clouds of solute atoms (Ananthakrishna and Sahoo 1981; Ananthakrishna and Valsakumar 1982; Valsakumar and Ananthakrishna 1983). The corresponding densities are denoted by $\tilde{\rho}_{m}(t), \tilde{\rho}_{i m}(t)$ and $\tilde{\rho}_{i}(t)$ respectively. Using some well known dislocation mechanisms, we set up rate equations for the densities of the dislocations. Further details of the model can be found in the above references. These equations are then coupled to the machine equation for the rate of change of stress. There are several parameters in the model corresponding to the rate constants of the transformations. One parameter that must be mentioned is the exponent $m$ which appears in the dependence of the velocity of dislocations on stress:

$$
v(\sigma)=v_{0}\left(\sigma / \sigma_{0}\right)^{m},
$$

where $\nu_{0}$ and $\sigma_{0}$ are constants. Another physically interesting drive parameter as a function of which the phenomenon occurs is the applied strain rate. There is a range of values of the parameters for which the steady state is unstable. Most of the analysis is carried out by keeping the parameter values within the instability domain and using the scaled strain rate $e$ as the drive parameter. As $e$ varied across the lower critical value, the behaviour changes from the normal yield to multiple yield. Simultaneously, we find that the negative SRS property sets in. The model also predicts an upper and a lower critical value for $e$ and the scaled concentration of the solute atoms for the existence of the phenomenon. We shall refer to this model as a dynamical model (DM).

The above model exhibits chaos and has a rich variety of behaviour. There are several physically relevant variables such as the scaled strain rate, the velocity exponent, the concentration of solute atoms etc. Here, we summarize the chaotic behaviour exhibited by the model (Ananthakrishna and Valsakumar 1983; Ananthakrishna 1990, 1992a) when the strain rate is varied. The chaotic behaviour is seen in a window of intermediate values of the strain rates $\sim 10^{-4} \mathrm{sec}^{-1}$ (Ananthakrishna 1990, 1992a). A plot of the noisy sequence of $\rho_{m}$ when the value of scaled strain rate $e=184$ well inside the chaotic regime is shown in figure 1. It is clear that there is no detectable order and the plot looks completely random. However, this apparent randomness arises from a set of deterministic differential equations. In such a case, given the exact initial conditions, there is no uncertainty in predicting the future. However, given two orbits, even the smallest amount of indeterminancy in the initial conditions very soon explodes at a exponential rate rendering predictability of the future of the two orbits impossible. This sensitivity to initial conditions is a characteristic feature of chaotic systems and is quantified by Lyapunov exponent, which describes the rate of divergence of nearby orbits. There is an order in this chaotic behaviour which manifests in the form of self-similarity of the strange attractor in the phase space of the variables. Such a phase plot of $\rho_{m}$ vs $\rho_{i m}$ showing a strange attractor is displayed in figure 2 . Quantifying this self-similar structure distinguishes it from the stochastic noise 


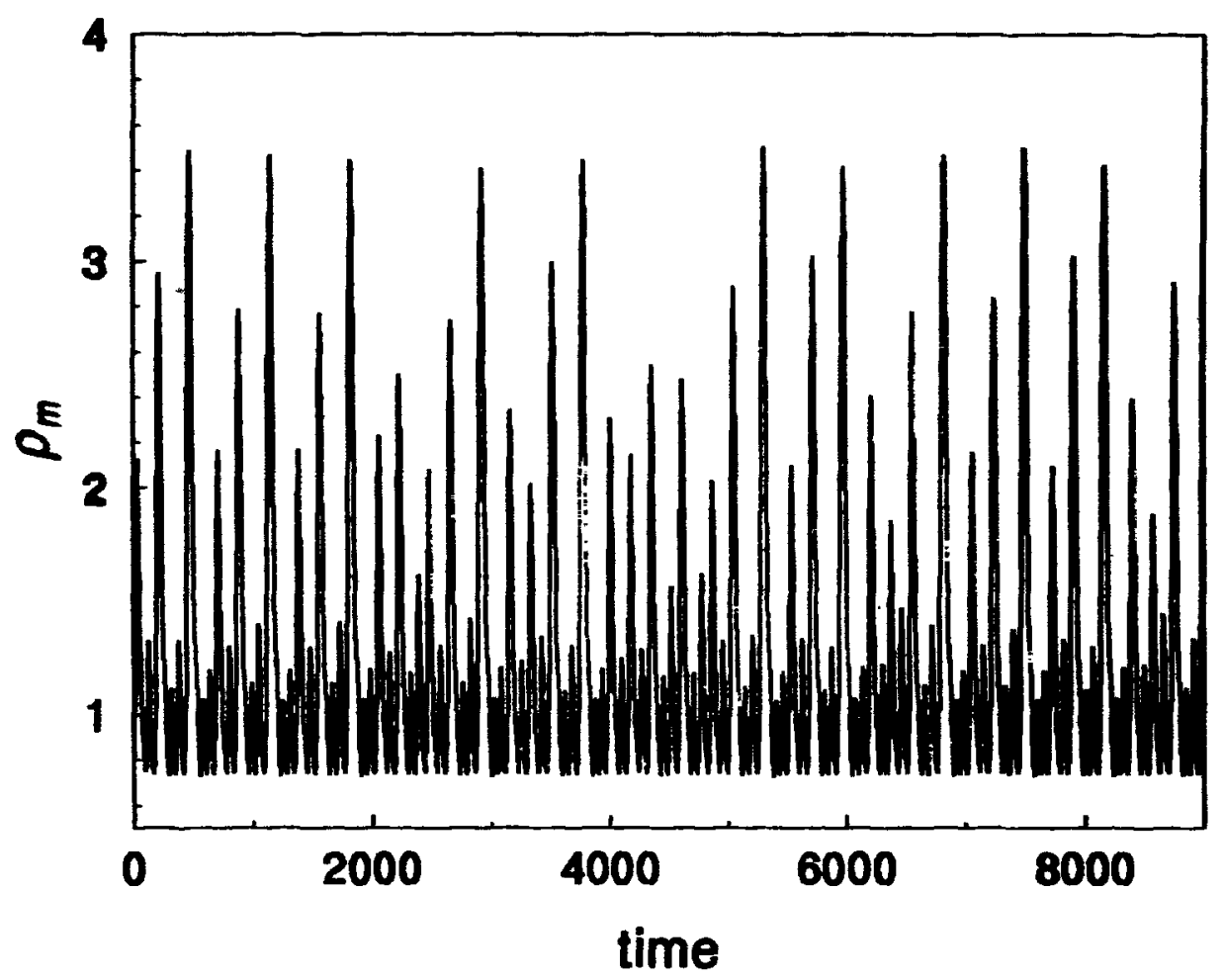

Fizure 1. A chasolic plot of $\rho_{m}$ as a function of time.

arising from many degrees of freedom. As a function of the applied strain rate, the model exhibits period doubling and period halving (backward) sequences. This rate of convergence of the period doubling sequence as a function of the drive parameter $e$, is measured by a quantity called Feigenbaum's exponent. When $m=2$, we find the Feigenbaum exponent $\delta=4.66$, which is the same as that for the one dimensional quadratic map (Feigenbaum 1978). This also means that the Poincare retum map of this system has a quadratic maximum in all the four variables. One such map constructed by plotting $\phi(n+1)$ vs $\phi(n)$, where $\phi(n)$ is the peak value of the scaled stress at time $n$, is shown in figure 3 . The nature of convergence of the period doubling and the period halving sequences is sensitive to the velocity exponent $m$. For instance, it exhibits a bubble structure for values of $m>2$. The model predicts that the chaotic behaviour is seen only in a window of strain rates in which the PLC effect is observed. Since no experiment is free of noise inherent in the measuring instrument, one always finds that both types of signals are superposed on each other. There are a number of methods developed in the literature with varying degrees of sophistication which helps us distinguish the two different types of time series (Grassberger and Procaccia 1983; Wolf et al 1985; Broomhead and King 1986; Albano et al 1988). It is also possible to cstimate the extent of stochastic noise superposed on the deterministic noise. Using one such method we were able to analyze two different sets of experimental signals. 


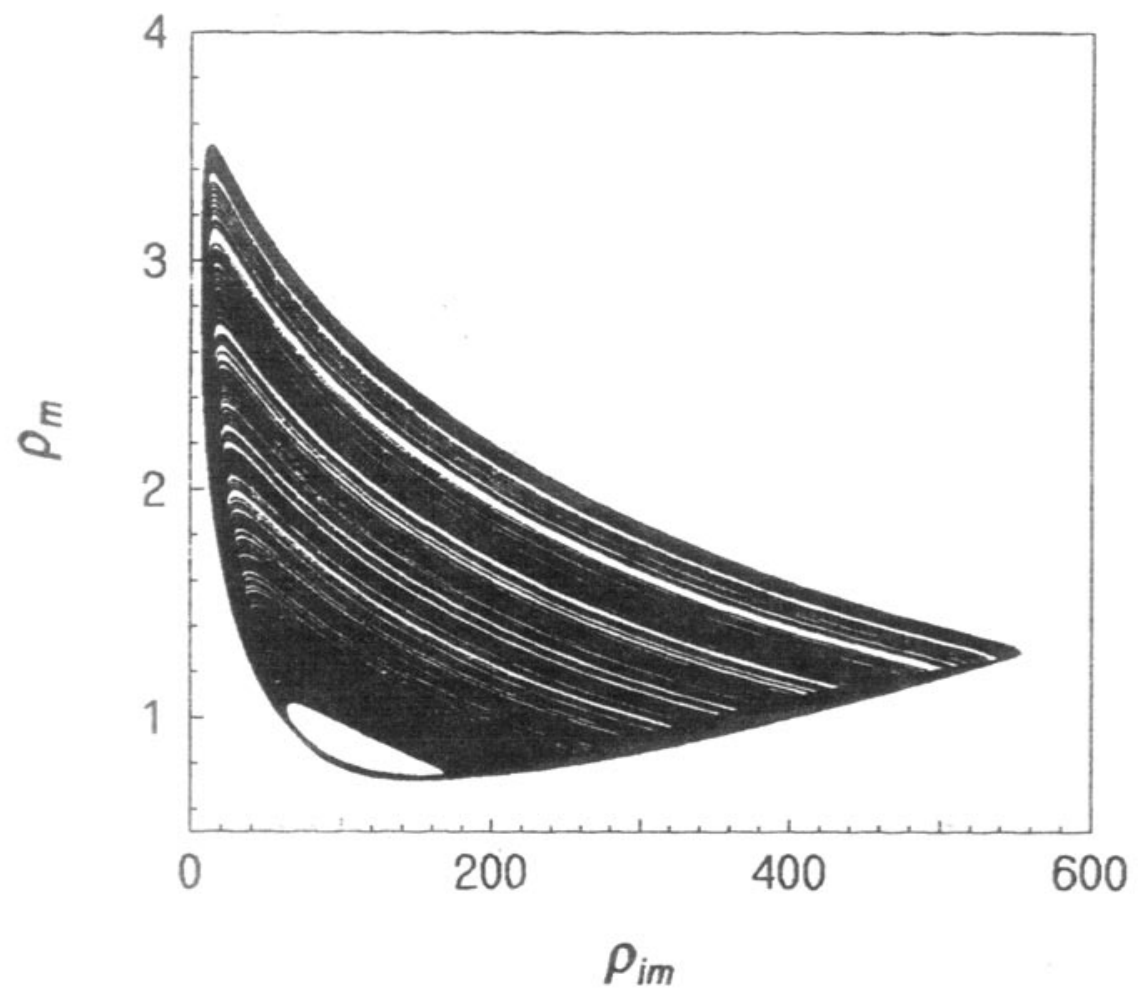

Pisce 2 A phase plot of the scaled mobile dislocation density $\rho_{\text {a }}$ ws the immobile descity $p_{\text {. }}$, showing a self-similar structure.

The analysis showed that experimental signals of stress were indeed due to deterministic noise (Ananthakrishna et al 1992, 1994; Ananthakrishna 1993). This means that the prediction of the theory is correct. This also means that a correct description of the phenomenon should have a dynamical basis. In addition to this we could infer the following. As mentioned in the introduction the experimental sample is a spatially inhomogeneous and any spatially extended system has infinite degrees of freedom. Yet the signals show that the system behaves as if there are only a few degrees of freedom. Thus, these modes should correspond to collective degrees of freedom. We shall make use of this aspect in constructing a coupled lattice map for the description of the spatial aspect of jerky flow.

As mentioned in the introduction, devising an acceptable scheme for description of the formation and propagation of bands has been a controversial aspect. Inspite of this, several models exist including our own attempt which involves extending the above model by introducing simple gradient terms for the dislocation densities. Thus, the starting point is just a set of continuity equations for the dislocation densities coupled to the machine equation which acts as a equation of constraint. For detail of this extended dynamical model (EDM) see Ananthakrishna (1993). Here we wish to emphasize that even with such a simple spatial term, EDM predicts the formation and propagation of dislocation bands. The velocity of the 


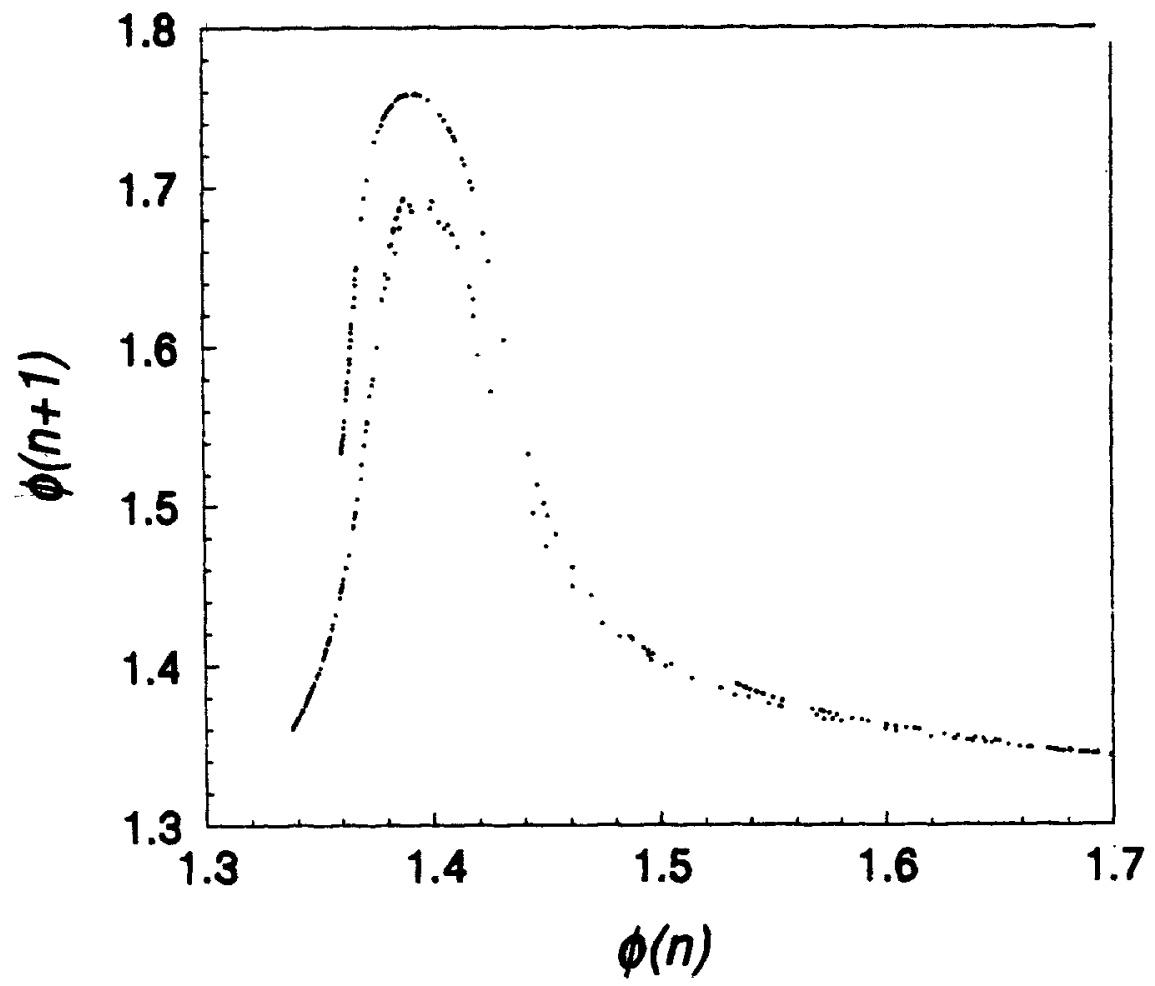

Figure 3. One dimensional map corresponding to the scaled stress $\phi$.

band predicted by EDM is consistent with the only experimental measurement available in the literature (Chihab et al 1987).

\section{A coupled map lattice model for the jerky now}

The method we follow here is to set up a coupled lattice map. Here one provides appropriate spatial coupling between the individual chaotic elements. In general such models are comparatively simple, yet they exhibit rich dynamics. In attempting to device such a model, it would be desirable to preserve the basic features of the two models (DM and EDM). We will extract the pertinent points for devising a simple enough model compared to the two models summarized above. Such an attempt is certainly desirable, since the above models consist of coupled set of nonlinear differential equations and are difficult to analyze. We will use the following two aspects of DM and EDM. First, we will use the fact that all the four variables namely the three dislocation densities and the stress have a Poincare return map with a quadratic maximum. Second, we note that we introduced simple gradient terms into EDM as an additional feature. The first point implies that we could assume that each spatial element is behaving in a chaotic way. We further note that the quadratic nature of the Poincare maps of each of the variables is a result of nonlinear evolution equations. Second point implies that neighbouring elements 
should be coupled using a first difference. Thus, to simplify the model even further we use just one dislocation density and the physically interesting density is the mobile dislocation density. (This limits the extent to which we can interpret the results as we will see later.) We note further that the values of the variables we encounter in the one dimensional maps are restricted to the domain $[0,1]$. Thus, all the variables in the coupled lattice model corresponding to the old variables should be scaled appropriately so that their values are in this domain. We shall denote the corresponding density by $x(n, i)$, where $n$ refers to the time index and $i$ refers to the spatial position on a one dimensional lattice. Recall that in EDM, we have a term $\nabla\left(v(\sigma) \rho_{m}\right)$, in the continuity equation for the mobile density. Here the velocity of dislocations are functions of the applied stress $\sigma$. This will be function of time alone. We will denote the scaled stress by $\phi(n)$. We further recall that the phenomenon occurs as a function of the drive parameter, namely, the applied strain rate and this parameter appears in the stress equation only. This means that the parameter that we will introduce the map for $\phi(n)$ should be taken to correspond to the applied strain rate scaled appropriately, which we will denote by $s$ (in such a way that its range is from 0 to 4). With these observations, we can write down the simplest form of a coupled lattice map as follows.

$$
\begin{aligned}
& x(n, i)=p r f(x(n, i))+(1-p)\left[s \phi_{n}\right]^{2}[f(x(n, i-1))-f(x(n, i))], \\
& \phi(n+1)=s f(\phi(n)), \\
& f(y)=y[1-y] .
\end{aligned}
$$

The velocity of dislocations as stated earlier is some power of the stress. For this reason we have introduced the term $[s \phi(n)]^{2}$ as a multiplicative term in the gradient of $x(n, i)$. Note that the exponent used then corresponds to $m=2$. (Recall that it is for this case that we get a quadratic Poincare map.) The parameter $r$ is a control parameter for the quadratic map corresponding to $x(n, i)$. From DM we know that $r$ should be a function of applied strain rate apart from other parameters. Since we do not know the exact dependence, we will introduce it as a tuning parameter as a function of which the period doubling bifurcation arises. It is conventional to introduce an additional parameter $p$ to signify the on-site strength and $(1-p)$ to the near neighbour coupling (Kaneko 1989).

\section{Results and discussion}

The time evolution of the system is studied by preparing the system with random initial conditions for $x(0, i)$ and nonzero but small value for $\phi(0)$. We impose periodic boundary conditions on the lattice. For most part of our computations we have used a lattice size of 200 points. The values of the parameters used are $r=s=3.8$ and $p=0.1$. This means that the on-site term gives period two (because, $r \cdot p=3.42$ which falls in the domain of period two) for the dislocation density, but keeps the stress in the chaotic zone. In a short time, a very heterogeneous structure develops, with high and low value for the dislocation density as a function of spatial position. A typical early configuration for $n=60$ is shown in figure $4 a$. Figures $4 \mathrm{~b}-\mathrm{e}$ show the configurations at various times. As time progresses, we 

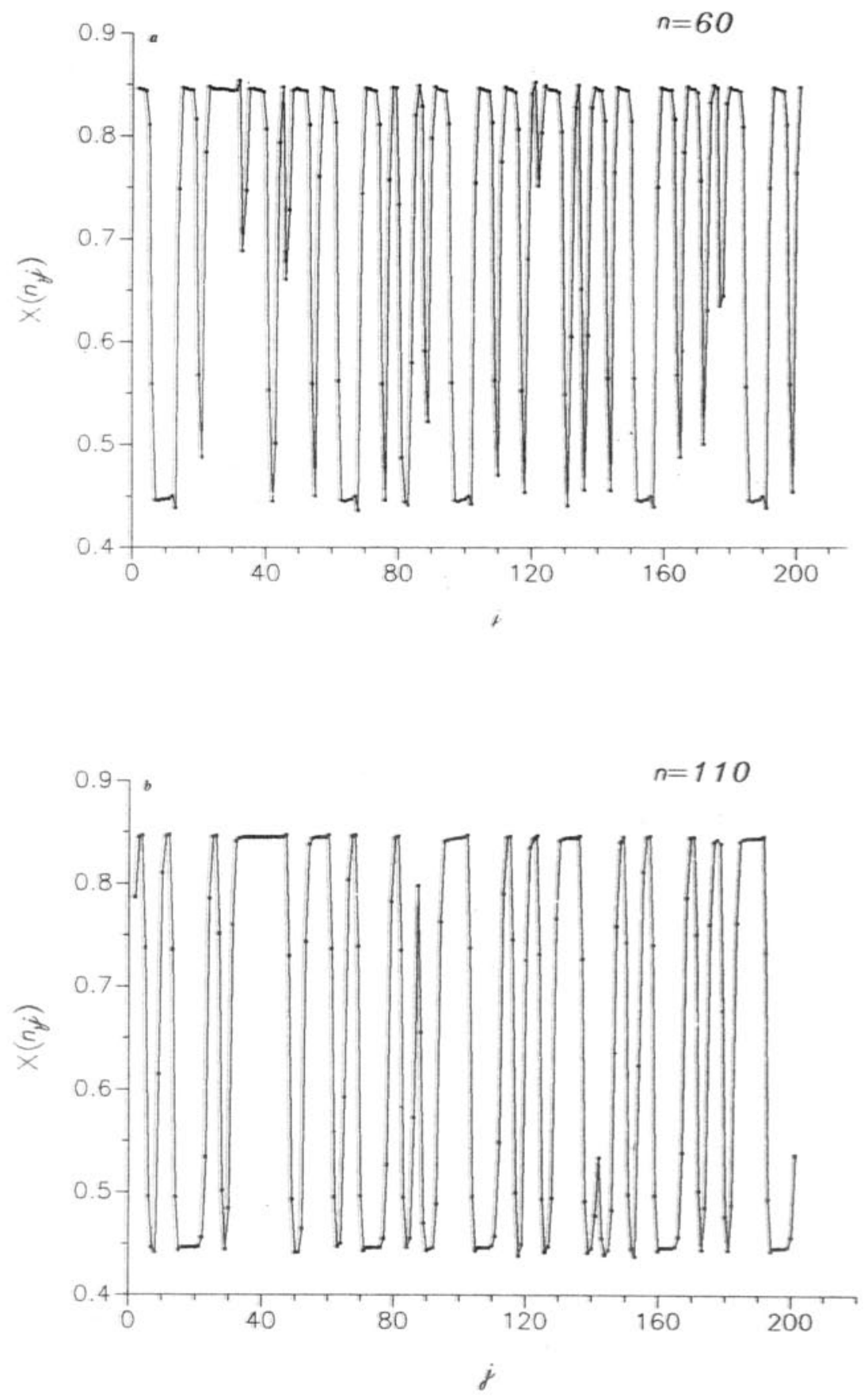

Fine the For coption, soe p. The 

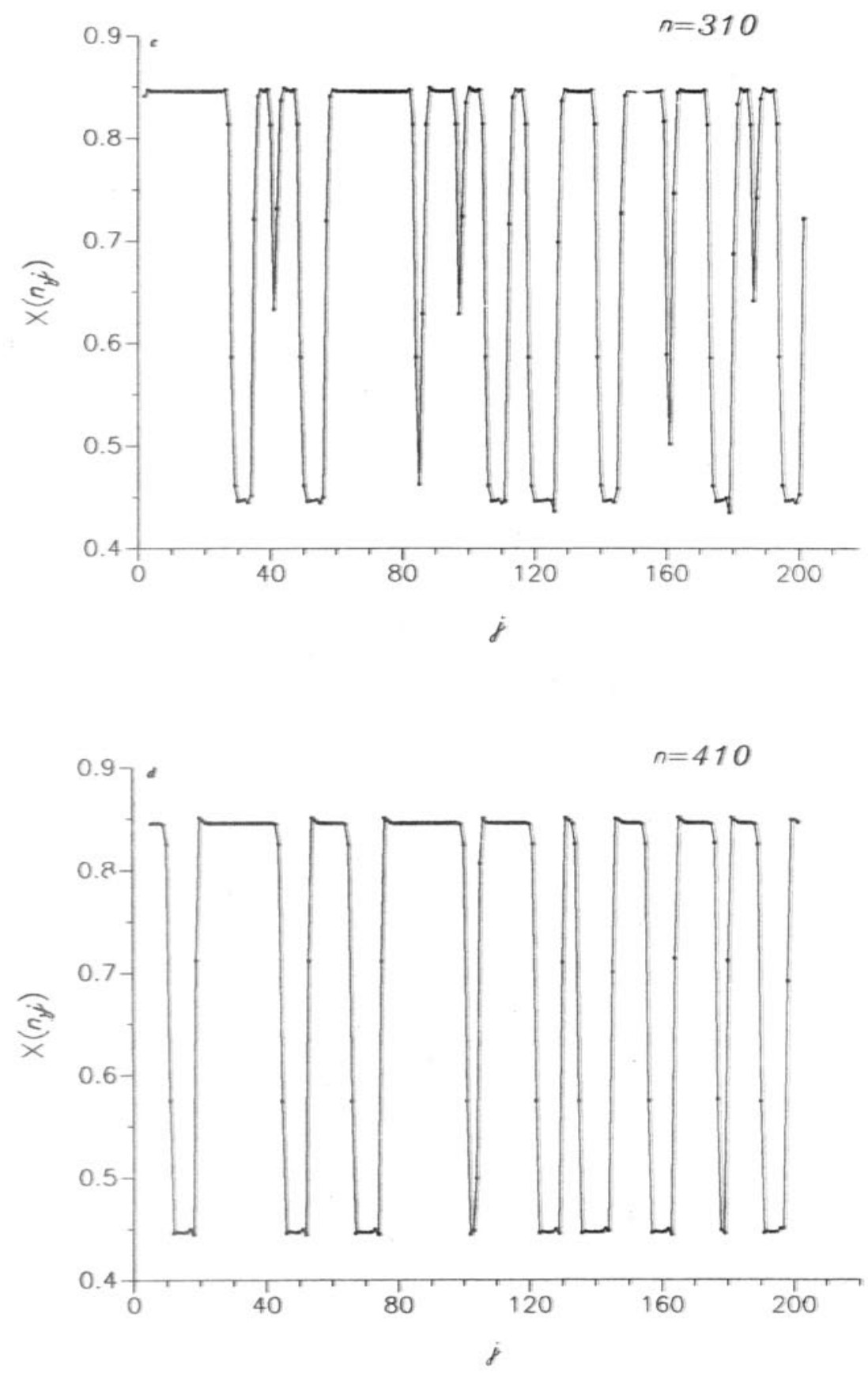

Figne ted For coption, soe p 780 


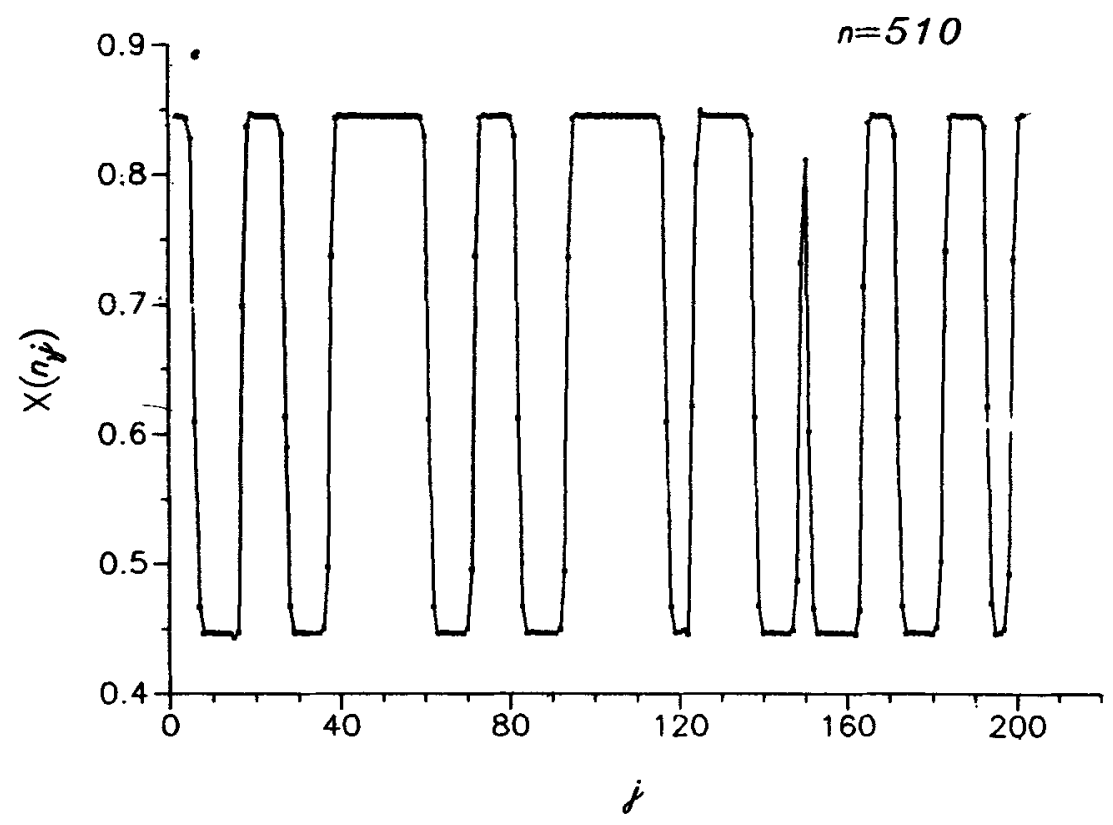

Figure 4. a. Dislocation density at various spatial points at an early stage of simulation $(n=60)$, b. dislocation density at various spatial points at a fairly early stage of simulation $(n=110)$, c. dislocation density at various spatial points at a intermediate time $(n=310)$, d. dislocation density at various spatial points at a late stage $(n=410)$ and e. dislocation density at various spatial points at a late stage $(n=510)$.

generally see two types of changes. In the early part of the simulation, we see that the width of the local bands with high and low dislocation densities are generally increasing. This can be seen by comparing the configuration of $n=60$ with $n=110$. We see that the bands with high and low dislocation densities have expanded by suppressing the rapid fluctuations. This is what one would expect physically in a realistic situation also. Second feature is that at later stages we see that in general the bands with high dislocation densities are expanding into the regions of low dislocation density. This can be seen by comparing the configurations at $n=410$ and $n=510$. Again, one should expect that this general feature is correct, since if we regard that low mobile dislocation density corresponds to no other type of dislocations, this would amount to less strain and one should expect that the front of the dislocation bands move into a region of lower strain. It must be remarked here that this is a consequence of the simplicity of the model, since, it is possible that low mobile dislocation density could correspond to high immobile or forest dislocations. At later stages, for example, an attempt to overlay the configurations with most parts overlapping (some will not, for reasons that will be stated below), shows that the system as a whole 'moves' forward. For instance, if the first broad band of high $x$ value from $i=20$ to 42 at time $n=410$, is taken to correspond to the first broad band at $i=40$ at a time $n=510$, we see that the system as a whole appears to 'move' forward. (Note that the sites from $\mathbf{4 8 0}$ 
onward at $n=410$ then correspond to the sites from 0 to 20 due to the periodic boundary conditions.) Although, this might give the impression of new bands being created at the grips which once again pass into the sample, one does not know if this can be taken as a proper interpretation. One other aspect that need to be studied is the effect of the chaotic behaviour of the stress equation. In principle, it should induce newer and newer pulses in to the spatial part. This should induce new bands created at random. We have not examined this aspect in detail. It must be remarked here that we have presented the simplest coupled lattice model. It is possible to develop more realistic models. Further work and perhaps evolving better coupled lattice map models are worth examining. Such attempts are in progress.

\section{Acknowledgement}

The author wishes to acknowledge support from IFCPAR, grant no. 1108-1.

\section{References}

Albano A M, Muench J, Schwartz C, Mees A I and Rapp P E 1988 Phys. Rev. A38 3017

Ananthakrishna G and Sahoo D 1981 J. Phys. D4 2091

Ananthakrishna G and Valsakumar M C 1982 J. Phys. D15 L171

Ananthakrishna G and Valsakumar M C 1983 Phys. Lett. A95 69

Ananthakrishna G 1990 in Directions in chaos (ed.) Hao Bai-lin (Singapore: World Scientific) p. 133

Ananthakrishna G 1992a in Nonlinear phenomena in materials science-Vol. II (eds) G Martin and L P Kubin, Solid State Phenomena Vol 23 \& 24 (Switzerland: Trans Tech) p. 417

Ananthakrishna G 1992b Plenary Lecture, International conference on plasticity of materials; fundamental aspects of dislocation interactions, Ascona, Switzerland

Ananthakrishna G, Grossbras M, Vergnol J, Bouchaud E, Planes J and Kubin L P 1992 International conference on plasticity of materials: fundamental aspects of dislocation interactions, Ascona. Switzerland

Ananthakrishna G 1993 Scr. Metall. 291183

Ananthakrishna G et al 1994 Scr. Metall. (accepted)

Berge P, Pomeau Y and Vidal P 1984 Order in chaos (New York: John Wiley)

Broomhead D S and King G P 1986 Physica D20 217

Chihab K, Estrin Y, Kubin L P and Vergnol J 1987 Scr. Metall. 21203

Feigenbaum M J 1978 J. Stat. Phys. 1925

Grassberger P and Procaccia I 1983 Physica D9 189

Hao Bai-lin (ed.) 1988, 1989, 1990 Directions in chaos (Singapore: World Scientific) Vol. I-III

Jeanclaude V and Fressengens C 1993 C. R. Acad. Sci. Paris 315867

Kaneko K 1989 Physica D37 60

Kubin L P and Estrin Y 1985 Acta Metall. 362702

Kubin L P and Estrin Y 1990 Acta Metall. 38697

Kubin L P, Estrin Y and Aifantis E C (eds) 1993 Viewpoint set $* 21$; Scr. Metall. 29

Valsakumar M C and Ananthakrishna G 1983 J. Phys. D16 1055

Wolf A. Swift.J B, Swinney H L and Vastano J A 1985 Physica D16 285

Zbib $H$ M and Aifantis E C 1988 Scr. Metall. 22133 